\title{
Microwave magnetoelectric effect in structures based on ferromagnetic metals
}

\author{
Alexander Tatarenko ${ }^{1, *}$, Oleg Sokolov ${ }^{1}$, Sergey Ivanov ${ }^{1}$, Mirza Bichurin ${ }^{1}$, and Yaojin Wang $^{2}$ \\ ${ }^{1}$ Novgorod State University, Veliky Novgorod,173020, Russia \\ ${ }^{2}$ Nanjing University of Science and Technology, 200 Xiaolingwei, Nanjing, China
}

\begin{abstract}
The results of a theoretical calculation of the resonant magnetizing field values for ferromagnetic resonance in thin films of nickel, iron and cobalt for frequencies of $3,10,30 \mathrm{GHz}$ for various orientations of this field are presented. Analytical dependences of the ferromagnetic line shifts on the electric field strength are obtained for twolayer magnetoelectric composites in which PZT, PMN-PT or PZN-PT disks are used as piezoelectrics on which thin films of $\mathrm{Ni}, \mathrm{Fe}$ or $\mathrm{Co}$ are deposited.
\end{abstract}

\section{Introduction}

In connection with the development of spintronics, interest in the magnetoelectric (ME) effect in nanostructures has increased. The ME effect in multiferroics in the terahertz range based on various manganites has recently been studied [1]. It is of interest to study the magnetoelectric effect in the ferromagnetic resonance (FMR) region in nanostructures based on ferromagnetic metals [2]. This paper presents the results of studies of the features of FMR in thin films of nickel, iron, and cobalt (film thickness $\sim 50 \mathrm{~nm}$ ). The objects of theoretical research were two-layer ME composites, in which PZT, PMN-PT or PZN-PT disks with a thickness of $0.5 \mathrm{~mm}$ was used as piezoelectric materials, on which thin films of $\mathrm{Ni}, \mathrm{Fe}$ or $\mathrm{Co}$ were deposited. The values of the resonance bias field for frequencies of 3, 10, $30 \mathrm{GHz}$ with different orientations of this field are calculated. Cases are considered when the magnetizing field is perpendicular to the plane of a thin metal film and also directed in the plane of a thin metal film along or perpendicular to easy magnetization axis. The main attention is paid to the theoretical study of the FMR line shift in two-layer ME composites based on thin films of ferromagnetic metals under the influence of an electric field.

\subsection{Calculation methods of the FMR line shift in two-layer magnetoelectric composites}

The calculation method for the FMR line shifting in two-layer ME composites consists of two stages. First, the values of the resonant magnetizing field are calculated at frequencies of $3,10,30 \mathrm{GHz}$ for various orientations of this field. So, cases were considered when the magnetizing field is perpendicular to the plane of a thin metal film, and also directed in the

*Corresponding author: alexandr.tatarenko@novsu.ru 
plane of a thin metal film along or perpendicular to the axis of easy magnetization. Then, two-layer ME composites based on thin films of ferromagnetic metals $\mathrm{Ni}, \mathrm{Fe}$ or $\mathrm{Co}$ are examined. In case when a constant electric field directed perpendicular to the plane of the disk to influence at ME composites, the FMR line shifts as a result of the microwave ME effect [3]. The calculated dependences of the FMR line shift on the electric field strength were obtained by using the method of effective demagnetizing factors.

The use of layered structures based on nanofilms of ferromagnetic metals will allow to proceed to further miniaturization of devices and design a number of new nanoelectronic microwave devices [4].

\section{FMR in a ferromagnetic metal nanofilm}

Let us consider a ferromagnetic metal nanofilm to which a constant magnetizing field is applied perpendicular to the plane of the film. We assume that the magnitude of this field is sufficiently large and the ferromagnetic metal film is uniformly magnetized to saturation. We direct the axis 3 along $H_{0}$, then $H_{0}$ has components $\left(0,0, H_{0}\right)$, and the equilibrium magnetization has components $\left(0,0, M_{0}\right)$. Axis 1 is directed along the axis of easy magnetization of the nickel (iron) film.

The equation of the motion of magnetization in a ferromagnetic metal nanofilm under the action of a high-frequency magnetic field $h$ in the presence of a constant magnetic field $H$, taking into account dissipation is

$$
\frac{\partial \vec{M}}{\partial t}=\gamma\left[\vec{M} \times \frac{\partial W}{\partial \vec{M}}\right]+i \omega \alpha \frac{\left[\vec{M}_{0} \times \vec{m}\right]}{M_{0}}
$$

where the full magnetization consists of the equilibrium and high-frequency components

$$
\vec{M}=\vec{M}_{0}+\vec{m}
$$

and the density of free energy is

$$
W=-\vec{M} \cdot \vec{H}+\frac{1}{2 \mu_{0}} \sum N_{11}^{i} M_{1}^{2}+\frac{1}{2 \mu_{0}} \sum N_{22}^{i} M_{2}^{2}+\frac{1}{2 \mu_{0}} \sum N_{33}^{i} M_{3}^{2}+\frac{1}{\mu_{0}} \sum N_{12}^{i} M_{1} M_{2}
$$

Then we write the dissipative term component wise

$$
i \omega \alpha \frac{\left[\vec{M}_{0} \times \vec{m}\right]}{M_{0}}=\frac{i \omega \alpha}{M_{0}}\left|\begin{array}{ccc}
\vec{i} & \vec{j} & \vec{k} \\
0 & 0 & M_{0} \\
m_{1} & m_{2} & m_{3}
\end{array}\right|=\frac{i \omega \alpha}{M_{0}}\left(-\vec{i} M_{0} m_{2}+\vec{j} M_{0} m_{1}\right)=i \omega \alpha\left(-\vec{i} m_{2}+\vec{j} m_{1}\right)
$$

The magnetic field strength also consists of two components

$$
\vec{H}=\vec{H}_{0}+\vec{h}
$$

Then we find the derivatives of free energy with respect to the components of magnetization

$$
\begin{aligned}
& \frac{\partial W}{\partial M_{1}}=-h_{1}+\frac{1}{\mu_{0}} \sum N_{11}^{i} M_{1}+\frac{1}{\mu_{0}} \sum N_{12}^{i} M_{2} \\
& \frac{\partial W}{\partial M_{2}}=-h_{2}+\frac{1}{\mu_{0}} \sum N_{22}^{i} M_{2}+\frac{1}{\mu_{0}} \sum N_{12}^{i} M_{1} \\
& \frac{\partial W}{\partial M_{3}}=-H_{0}-h_{3}+\frac{1}{\mu_{0}} \sum N_{33}^{i} M_{3}
\end{aligned}
$$

Next, let's us write the equation of motion for the components 


$$
\begin{aligned}
& i \omega m_{1}=\gamma\left[m_{2} \frac{\partial W}{\partial M_{3}}-\left(M_{0}+m_{3}\right) \frac{\partial W}{\partial M_{2}}\right]-i \alpha \omega m_{2} \\
& i \omega m_{2}=\gamma\left[\left(M_{0}+m_{3}\right) \frac{\partial W}{\partial M_{1}}-m_{1} \frac{\partial W}{\partial M_{3}}\right]+i \alpha \omega m_{1} \\
& i \omega m_{3}=\gamma\left[m_{1} \frac{\partial W}{\partial M_{2}}-m_{2} \frac{\partial W}{\partial M_{1}}\right]
\end{aligned}
$$

Linearizing these equations and preserving only the first-order terms of smallness in them, we obtain a system of two linear inhomogeneous equations for two unknowns $m_{1}, m_{2}$

$$
\begin{aligned}
& \left(i \omega+\gamma \frac{M_{0}}{\mu_{0}} \sum N_{12}^{i}\right) m_{1}+\left[\gamma\left(H_{0}+\left\{\sum N_{22}^{i}-\sum N_{33}^{i}\right\} \frac{M_{0}}{\mu_{0}}\right)+i \alpha \omega\right] m_{2}=\gamma M_{0} h_{2} \\
& {\left[\gamma\left(H_{0}+\left\{\sum N_{11}^{i}-\sum N_{33}^{i}\right\} \frac{M_{0}}{\mu_{0}}\right)+i \alpha \omega\right] m_{1}+\left(-i \omega+\gamma \frac{M_{0}}{\mu_{0}} \sum N_{12}^{i}\right) m_{2}=\gamma M_{0} h_{1}}
\end{aligned}
$$

Having solved this system, we can write the form of the high-frequency magnetic susceptibility tensor

$$
\chi=\left(\begin{array}{ccc}
\chi_{11} & \chi_{12} & 0 \\
\chi_{21} & \chi_{22} & 0 \\
0 & 0 & 0
\end{array}\right)
$$

Where components of this tensor are

$$
\begin{aligned}
& \chi_{11}=\frac{-\gamma M_{0}\left[\gamma\left(H_{0}+\left\{\sum N_{22}^{i}-\sum N_{33}^{i}\right\} \frac{M_{0}}{\mu_{0}}\right)+i \alpha \omega\right]}{D} \\
& \chi_{22}=\frac{-\gamma M_{0}\left[\gamma\left(H_{0}+\left\{\sum N_{11}^{i}-\sum N_{33}^{i}\right\} \frac{M_{0}}{\mu_{0}}\right)+i \alpha \omega\right]}{D} \\
& \chi_{12}=\chi_{s}-i \chi_{a} \\
& \chi_{21}=\chi_{s}+i \chi_{a} \\
& \chi_{s}=\frac{\gamma^{2} M_{0}^{2}}{\mu_{0} D} \sum N_{12}^{i} \\
& \chi_{a}=\frac{\gamma M_{0} \omega}{D}
\end{aligned}
$$

and

$$
\begin{aligned}
& D=\omega^{2}\left(1+\alpha^{2}\right)-\omega_{0}^{2}-i \gamma \alpha \omega\left(2 H_{0}+\left\{\sum N_{11}^{i}+\sum N_{22}^{i}-2 \sum N_{33}^{i}\right\} \frac{M_{0}}{\mu_{0}}\right) \\
& \omega_{0}^{2}=\gamma^{2}\left(H_{0}+\left\{\sum N_{11}^{i}-\sum N_{33}^{i}\right\} \frac{M_{0}}{\mu_{0}}\right)\left(H_{0}+\left\{\sum N_{22}^{i}-\sum N_{33}^{i}\right\} \frac{M_{0}}{\mu_{0}}\right)-\gamma^{2}\left(\sum N_{12}^{i} \frac{M_{0}}{\mu_{0}}\right)^{2}
\end{aligned}
$$

We will be interested in the imaginary parts of the complex components of highfrequency susceptibility $\chi_{11}^{\prime \prime}, \chi_{22}^{\prime \prime}, \chi_{a}^{\prime \prime}$ 


$$
\begin{aligned}
& \chi_{11}^{\prime \prime}=-\alpha \gamma M_{0} \omega \frac{\omega^{2}\left(1+\alpha^{2}\right)-\omega_{0}^{2}+\gamma^{2}\left(H_{0}+\left\{\sum N_{22}^{i}-\sum N_{33}^{i}\right\} \frac{M_{0}}{\mu_{0}}\right)\left(2 H_{0}+\left\{\sum N_{11}^{i}+\sum N_{22}^{i}-2 \sum N_{33}^{i}\right\} \frac{M_{0}}{\mu_{0}}\right)}{\left[\omega^{2}\left(1+\alpha^{2}\right)-\omega_{0}^{2}\right]^{2}+\gamma^{2} \alpha^{2} \omega^{2}\left(2 H_{0}+\left\{\sum N_{11}^{i}+\sum N_{22}^{i}-2 \sum N_{33}^{i} \frac{M_{0}}{\mu_{0}}\right)\right.} \\
& \chi_{22}^{\prime \prime}=-\alpha \gamma M_{0} \omega \frac{\omega^{2}\left(1+\alpha^{2}\right)-\omega_{0}^{2}+\gamma^{2}\left(H_{0}+\left\{\sum N_{11}^{i}-\sum N_{33}^{i}\right\} \frac{M_{0}}{\mu_{0}}\right)\left(2 H_{0}+\left\{\sum N_{11}^{i}+\sum N_{22}^{i}-2 \sum N_{33}^{i}\right\} \frac{M_{0}}{\mu_{0}}\right)}{\left[\omega^{2}\left(1+\alpha^{2}\right)-\omega_{0}^{2}\right]^{2}+\gamma^{2} \alpha^{2} \omega^{2}\left(2 H_{0}+\left\{\sum N_{11}^{i}+\sum N_{22}^{i}-2 \sum N_{33}^{i}\right\} \frac{M_{0}}{\mu_{0}}\right)} \\
& \chi_{a}^{\prime \prime}=\frac{\alpha \gamma^{2} M_{0} \omega^{2}\left(2 H_{0}+\left\{\sum N_{11}^{i}+\sum N_{22}^{i}-2 \sum N_{33}^{i}\right\} \frac{M_{0}}{\mu_{0}}\right)}{\left[\omega^{2}\left(1+\alpha^{2}\right)-\omega_{0}^{2}\right]^{2}+\gamma^{2} \alpha^{2} \omega^{2}\left(2 H_{0}+\left\{\sum N_{11}^{i}+\sum N_{22}^{i}-2 \sum N_{33}^{i}\right\} \frac{M_{0}}{\mu_{0}}\right)}
\end{aligned}
$$

Demagnetizing factors associated with sample shape

$$
\begin{aligned}
& N_{11}^{F}=N_{22}^{F}=0 \\
& N_{33}^{F}=1
\end{aligned}
$$

Demagnetizing factors associated with uniaxial magnetic anisotropy

$$
\begin{aligned}
& N_{11}^{a}=-\frac{\mu_{0} H_{a}}{M_{0}} \\
& N_{22}^{a}=N_{33}^{a}=0
\end{aligned}
$$

The resonant values of the constant magnetizing field $H_{0}$, found numerically in Maple for the microwave frequency $f=10 \mathrm{GHz}$ for the $\chi_{11}^{\prime \prime}, \chi_{22}^{\prime \prime}, \chi_{a}^{\prime \prime}$, coincide with each other to an accuracy of $1 \mathrm{~A} / \mathrm{m}$ and with the value found from the condition

$$
\omega^{2}=\omega_{0}^{2}
$$

In view of (10), from (14) we find the resonance value of the constant magnetizing field $H_{0}$

$$
H_{0}=\frac{\gamma M_{0}\left(2 \sum N_{33}^{i}-\sum N_{11}^{i}-\sum N_{22}^{i}\right)+\sqrt{\gamma^{2} M_{0}^{2}\left[\left(\sum N_{11}^{i}-\sum N_{22}^{i}\right)^{2}+4\left(\sum N_{12}^{i}\right)^{2}\right]+4 \mu_{0}^{2} \omega^{2}}}{2 \gamma \mu_{0}}
$$

Substituting (12), (13) into (15), we obtain the values of the constant magnetizing field for nickel $H_{0} \approx 4439,6850,13745$ Oe at resonant frequencies $f=3,10,30 \mathrm{GHz}$, respectively. For iron we obtain the values of the constant magnetizing field for nickel $H_{0} \approx 2414,4824,11720$ Oe at same frequencies, respectively.

We similarly consider the case when a constant magnetizing field is directed in the film plane along the axis of easy magnetization of the nickel (iron) film. For nickel, we have $H_{0} \approx 98,1973,8626$ Oe and for iron $H_{0} \approx 381,2672,9530$ Oe at the same frequencies, respectively.

For the case when a constant magnetizing field is directed in the plane of the film perpendicular to the axis of easy magnetization of the nickel (iron) film, For nickel, we have $H_{0} \approx 483,2317,8942$ Oe and for iron $H_{0} \approx 730,2986,9831$ Oe at the same frequencies, respectively.

For cobalt, there are only demagnetizing factors associated with the shape of the sample

$$
\begin{aligned}
& N_{11}^{F}=N_{22}^{F}=0 \\
& N_{33}^{F}=1
\end{aligned}
$$

Therefore, from (15) we have the constant magnetizing field for cobalt $H_{0} \approx 15500,17830,24497$ Oe at resonant frequencies $f=3,10,30 \mathrm{GHz}$, respectively. 
Similarly, we consider the case when the constant magnetizing field is directed in the plane of the cobalt film, and the values obtained are $H_{0} \approx 69,730,5102$ Oe at resonant frequencies $f=3,10,30 \mathrm{GHz}$, respectively.

\section{FMR line shift under an applied electric field}

First, we write (14) without taking into account the effective demagnetizing factors arising from the action of the electric field strength $E$

$$
\omega^{2}=\gamma^{2}\left(H_{0}+\left\{\sum_{i \neq E} N_{11}^{i}-\sum_{i \neq E} N_{33}^{i}\right\} \frac{M_{0}}{\mu_{0}}\right)\left(H_{0}+\left\{\sum_{i \neq E} N_{22}^{i}-\sum_{i \neq E} N_{33}^{i}\right\} \frac{M_{0}}{\mu_{0}}\right)-\gamma^{2}\left(\sum_{i \neq E} N_{12}^{i} \frac{M_{0}}{\mu_{0}}\right)^{2}
$$

Now we write (14), taking into account the small correction $\delta H_{E}$ associated with small effective demagnetizing factors arising from the action of the electric field strength $E$

$$
\begin{aligned}
& \omega^{2}=\gamma^{2}\left(H_{0}+\delta H_{E}+\left\{\sum_{i \neq E} N_{11}^{i}-\sum_{i \neq E} N_{33}^{i}+N_{11}^{E}-N_{33}^{E}\right\} \frac{M_{0}}{\mu_{0}}\right) \times \\
& \times\left(H_{0}+\delta H_{E}+\left\{\sum_{i \neq E} N_{22}^{i}-\sum_{i \neq E} N_{33}^{i}+N_{22}^{E}-N_{33}^{E}\right\} \frac{M_{0}}{\mu_{0}}\right)-\gamma^{2}\left(\left\{\sum_{i \neq E} N_{12}^{i}+N_{12}^{E}\right\} \frac{M_{0}}{\mu_{0}}\right)^{2}
\end{aligned}
$$

Subtract (17) from (18) and save in the obtained equation only the terms of the first order of smallness in $\delta H_{E}, N_{11}^{E}, N_{22}^{E}, N_{33}^{E}, N_{12}^{E}$

$$
\frac{M_{0}}{\mu_{0}} Q_{2}\left(N_{11}^{E}-N_{33}^{E}\right)+\frac{M_{0}}{\mu_{0}} Q_{3}\left(N_{22}^{E}-N_{33}^{E}\right)-\frac{M_{0}}{\mu_{0}} Q_{4} N_{12}^{E}+Q_{1} \delta H_{E}=0
$$

where

$$
\begin{aligned}
& Q_{1}=2 H_{0}+\left\{\sum_{i \neq E} N_{11}^{i}+\sum_{i \neq E} N_{22}^{i}-2 \sum_{i \neq E} N_{33}^{i}\right\} \frac{M_{0}}{\mu_{0}} \\
& Q_{2}=H_{0}+\left\{\sum_{i \neq E} N_{22}^{i}-\sum_{i \neq E} N_{33}^{i}\right\} \frac{M_{0}}{\mu_{0}} \\
& Q_{3}=H_{0}+\left\{\sum_{i \neq E} N_{11}^{i}-\sum_{i \neq E} N_{33}^{i}\right\} \frac{M_{0}}{\mu_{0}} \\
& Q_{4}=2 \sum_{i \neq E} N_{12}^{i} \frac{M_{0}}{\mu_{0}}
\end{aligned}
$$

From this condition we find the shift of the resonance line

$$
\delta H_{E}=\frac{M_{0}\left[Q_{2}\left(N_{33}^{E}-N_{11}^{E}\right)+Q_{3}\left(N_{33}^{E}-N_{22}^{E}\right)+Q_{4} N_{12}^{E}\right]}{\mu_{0} Q_{1}}
$$

Next, we find the demagnetizing factors for ferromagnetic metal/piezoelectric structures associated with the action of a constant electric field. Consider the case when a constant electric field is directed along axis 3.

We substitute the previously found demagnetizing factors (12) and (13) into (20)

$$
\begin{aligned}
& Q_{1}=2 H_{0}-H_{a}-\frac{2 M_{0}}{\mu_{0}} \\
& Q_{2}=H_{0}-\frac{M_{0}}{\mu_{0}} \\
& Q_{3}=H_{0}-H_{a}-\frac{M_{0}}{\mu_{0}} \\
& Q_{4}=0
\end{aligned}
$$

Formulas (22) are valid for nickel and iron, and in order to use them for cobalt it is necessary to put $H_{a}=0$ 
Consider the mechanical equilibrium of our structure. It is convenient to write the boundary conditions for a free sample in the form of a disk in cylindrical coordinates

$$
\begin{aligned}
& { }^{m} T_{3}={ }^{p} T_{3}=0 \\
& \left.{ }^{m} t^{m} T_{r r}\right|_{r=R}+\left.{ }^{p} t^{p} T_{r r}\right|_{r=R}=0 \\
& \left.{ }^{m} t^{m} T_{\theta \theta}\right|_{r=R}+\left.{ }^{p} t^{p} T_{\theta \theta}\right|_{r=R}=0
\end{aligned}
$$

where $R$ is the radius of the disk.

The components of the stress tensor in rectangular coordinates are expressed in terms of the components of the stress tensor in cylindrical coordinates as follows

$$
\begin{aligned}
& T_{1}=\cos ^{2}(\theta) T_{r r}+\sin ^{2}(\theta) T_{\theta \theta} \\
& T_{2}=\sin ^{2}(\theta) T_{r r}+\cos ^{2}(\theta) T_{\theta \theta}
\end{aligned}
$$

Multiplying (24) by $\cos ^{2}(\theta), \sin ^{2}(\theta)$ and using (25), we obtain

$$
\begin{aligned}
& \left.{ }^{m} t^{m} T_{1}\right|_{r=R}+\left.{ }^{p} t^{p} T_{1}\right|_{r=R}=0 \\
& \left.{ }^{m} t^{m} T_{2}\right|_{r=R}+\left.{ }^{p} t^{p} T_{2}\right|_{r=R}=0
\end{aligned}
$$

Given the quasistationary nature of the task, we can write

$$
\begin{aligned}
& { }^{m} t^{m} T_{1}+{ }^{p} t^{p} T_{1}=0 \\
& { }^{m} t^{m} T_{2}+{ }^{p} t^{p} T_{2}=0
\end{aligned}
$$

In view of (23), the material equations for the ferromagnetic phase

and for piezoelectric

$$
\begin{aligned}
& S_{1}={ }^{m} s_{11}{ }^{m} T_{1}+{ }^{m} s_{12}{ }^{m} T_{2} \\
& S_{2}={ }^{m} s_{12}{ }^{m} T_{1}+{ }^{m} s_{11}{ }^{m} T_{2}
\end{aligned}
$$

$$
\begin{aligned}
& S_{1}={ }^{p} S_{11}{ }^{p} T_{1}+{ }^{p} S_{12}{ }^{p} T_{2}+d_{31} E \\
& S_{2}={ }^{p} S_{12}{ }^{p} T_{1}+{ }^{p}{S_{11}}^{p} T_{2}+d_{31} E
\end{aligned}
$$

Solving together (27), (28) and (29), we find

$$
{ }^{m} T_{1}={ }^{m} T_{2}=\frac{d_{31}^{p} t E}{{ }^{p} t\left({ }^{m} s_{11}+{ }^{m} s_{12}\right)+{ }^{m} t\left({ }^{p} s_{11}+{ }^{p} s_{12}\right)}
$$

Part of the free energy associated with the presence of stresses arising from the action of an electric field on a piezoelectric

$$
\Delta W_{M E}=-\frac{3 \lambda_{s}}{2 M_{0}^{2}}\left({ }^{m} T_{1} M_{1}^{2}+{ }^{m} T_{2} M_{2}^{2}+{ }^{m} T_{3} M_{3}^{2}\right)
$$

From this conditions we find effective demagnetizing factors arising from the action of the electric field $E$

Then from (21) we find

$$
\begin{aligned}
& N_{11}^{E}=-\frac{3 \mu_{0} \lambda_{s}}{M_{0}^{2}}{ }^{m} T_{1} \\
& N_{22}^{E}=-\frac{3 \mu_{0} \lambda_{s}}{M_{0}^{2}}{ }^{m} T_{2} \\
& N_{33}^{E}=0 \\
& N_{12}^{E}=0
\end{aligned}
$$

$$
\delta H_{E}=\frac{3 \lambda_{s} d_{31}^{p} t E}{M_{0}\left[{ }^{p} t\left({ }^{m} s_{11}+{ }^{m} s_{12}\right)+{ }^{m} t\left({ }^{p} s_{11}+{ }^{p} s_{12}\right)\right]}
$$

The dependence of the FMR resonance line shift of the on the electric field is a direct proportion

where $\delta H_{E}$ is in Oe, and $E$ is in $\mathrm{kV} / \mathrm{cm}$.

$$
\delta H_{E}=k E
$$


The theoretical results of our calculations presented on the next Figures. Results for Ni deposited on PZT, PMN-PN and PZN-PT are shown on Fig. 1.

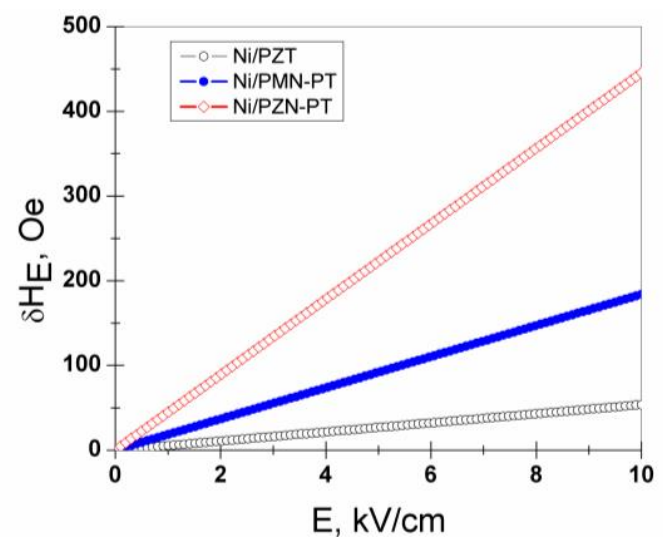

Fig. 1. Result of the FMR line shift calculation for composite structures of Ni/PZT, Ni/PMN-PT and $\mathrm{Ni} / \mathrm{PZN}-\mathrm{PT}$.

Results for Fe deposited on PZT, PMN-PN and PZN-PT are shown on Fig. 2.

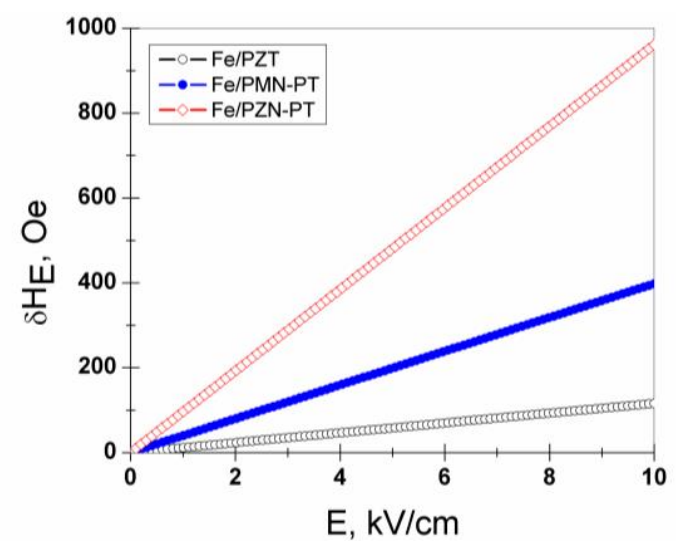

Fig. 2. Result of the FMR line shift calculation for composite structures of Fe/PZT, Fe/PMN-PT and $\mathrm{Fe} / \mathrm{PZN}-\mathrm{PT}$.

Results for Co deposited on PZT, PMN-PN and PZN-PT are shown on Fig. 3.

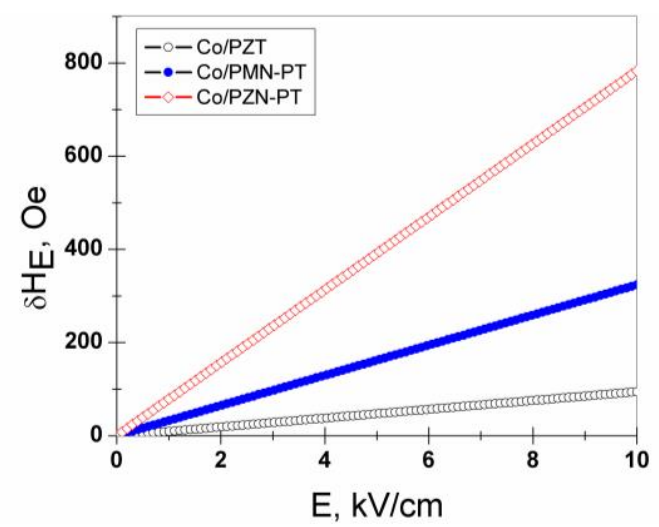

Fig. 3. Result of the FMR line shift calculation for composite structures of Co/PZT, Co/PMN-PT and Co/PZN-PT. 
Due to the specific symmetry of the problem, $k$ does not depend on the directivity of the magnetizing field and the magnitude of the resonant frequency $f$.

\section{Conclusion}

A calculation method for determining the FMR line shift in two-layer ME composites based on thin ferromagnetic metals films under the influence of an electric field is proposed.

Analytical dependences of the FMR line shift in such composites on the electric field strength are obtained.

This work was supported by the Russian Federation Grant RFBR 19-57-53001 NNSF_a.

\section{References}

1. A.A. Mukhin, et al., Phys. Usp. 52, 851 (2009)

2. E.A. Karashtin, A.A. Fraerman, Phys. Solid State 58, 2228 (2016)

3. M.I. Bichurin, V.M. Petrov, R.V. Petrov, A.S. Tatarenko, Magnetoelectric Composites (Pan Stanford Publ., 280 p., 2019)

4. T. Nan et al., Sci. Rep. 4, 3688 (2014) 